Keywords: colorectal- neoplasms/diagnosis; early detection of cancer; great britain/epidemiology; mass screening/methods; occult blood; patient selection; predictive value of tests; programme evaluation; quality of health care

\title{
Optimising faecal occult blood screening: retrospective analysis of NHS Bowel Cancer Screening data to improve the screening algorithm
}

\begin{abstract}
J Geraghty ${ }^{1,2}$, P Butler ${ }^{3}$, H Seaman ${ }^{3,4}$, J Snowball ${ }^{3}$, S Sarkar ${ }^{1}$, R Blanks ${ }^{5}$, S Halloran ${ }^{3,4}$, K Bodger ${ }^{2,6}$ and CJ Rees ${ }^{\star, 7,8,9}$
${ }^{1}$ Royal Liverpool University Hospital, Liverpool, UK; ${ }^{2}$ University of Liverpool, Liverpool, UK; ${ }^{3}$ Bowel Cancer Screening Southern Programme Hub, Guildford, UK; ${ }^{4}$ University of Surrey, Guildford, Surrey, UK; ${ }^{5}$ Cancer Epidemiology Group at the University of Oxford, Oxford, UK; ${ }^{6}$ Aintree University Hospital NHS Trust, Liverpool, UK; ${ }^{7}$ South of Tyne Bowel Cancer Screening Centre, South Tyneside District Hospital, South Shields, UK; ${ }^{8}$ Durham University School of Medicine, Pharmacy and Health, Durham, UK and ${ }^{9}$ on behalf of the Northern Region Endoscopy Group
\end{abstract}

Background: Colorectal neoplasia causes bleeding, enabling detection using Faecal Occult Blood tests (FOBt). The National Health Service (NHS) Bowel Cancer Screening Programme (BCSP) guaiac-based FOBt (gFOBt) kits contain six sample windows (or 'spots') and each kit returns either a positive, unclear or negative result. Test kits with five or six positive windows are termed 'abnormal' and the subject is referred for further investigation, usually colonoscopy. If $1-4$ windows are positive, the result is initially 'unclear' and up to two further kits are submitted, further positivity leads to colonoscopy ('weak positive'). If no further blood is detected, the test is deemed 'normal' and subjects are tested again in 2 years' time. We studied the association between spot positivity \% (SP\%) and neoplasia.

Methods: Subjects in the Southern Hub completing the first of two consecutive episodes between April 2009 and March 2011 were studied. Each episode included up to three kits and a maximum of 18 windows (spots). For each positivity combination, the percentage of positive spots out of the total number of spots completed by an individual in a single-screening episode was derived and named 'SP\%'. Fifty-five combinations of SP can occur if the position of positive/negative spots on the same test card is ignored. The proportion of individuals for whom neoplasia was identified in Episode 2 was derived for each of the 55 spot combinations. In addition, the Episode 1 spot pattern was analysed for subjects with cancer detected in Episode 2.

Results: During Episode 2, 284261 subjects completed gFOBT screening and colonoscopies were performed on 3891 (1.4\%) subjects. At colonoscopy, cancer was detected in 7.4\% $(n=286)$ and a further 39.8\% $(n=1550)$ had adenomas. Cancer was detected in $21.3 \%$ of subjects with an abnormal first kit (five or six positive spots) and in $5.9 \%$ of those with a weak positive test result. The proportion of cancers detected was positively correlated with SP\%, with an $R^{2}$ correlation (linear) of 0.89. As the SP\% increased from 11 to $100 \%$, so the colorectal cancer (CRC) detection rate increased from 4 to $25 \%$. At the lower SP\%s, from 11 to $25 \%$, the CRC risk was relatively static at $\sim 4 \%$. Above an SP\% of $25 \%$, every 10 -percentage points increase in the SP\%, was associated with an increase in cancer detection of $2.5 \%$.

Conclusions: This study demonstrated a strong correlation between SP\% and cancer detection within the NHS BCSP. At the population level, subjects' cancer risk ranged from 4 to $25 \%$ and correlated with the gFOBt spot pattern. Some subjects with an SP\% of $11 \%$ proceed to colonoscopy, whereas others with an SP\% of $22 \%$ do not. Colonoscopy on patients with four positive spots in kit 1 (SP\% 22\%) would, we estimate, detect cancer in 4\% of cases and increase overall colonoscopy volume by $6 \%$. This study also demonstrated how screening programme data could be used to guide its ongoing implementation and inform other programmes.

*Correspondence: Professor C Rees; E-mail: Colin.Rees@stft.nhs.uk

This work was submitted on behalf of the NHS Bowel Cancer Screening Evaluation Group.

Received 13 May 2014; revised 30 July 2014; accepted 7 August 2014; published online 16 September 2014

(C) 2014 Cancer Research UK. All rights reserved 0007 - 0920/14 
Colorectal cancer (CRC) is the second most common cancer in the United Kingdom, accounting for 41000 new cases and about 16000 deaths every year (Office for National Statistics., 2010). In the United Kingdom, the 5-year survival for CRC is around $54 \%$, which is significantly lower than countries with comparable wealth. In Australia, $66 \%$ of cases are alive at 5 years and in Sweden 63\% (Berrino et al, 2007; Coleman et al, 2011). The reason for this relatively poor survival is the late presentation of advanced stage cancer, which reduces the chance of curative treatment (Gatta et al, 2000; Coleman et al, 2011). The principle of bowel cancer screening is to detect cancer at a pre-symptomatic stage, leading to earlier diagnosis and improved clinical outcome (Bernie et al, 1998; Hewitson et al, 2007). Screening also enables the detection and excision of adenomas, thereby reducing CRC risk.

Following a review of all screening options, the National Health Service (NHS) adopted a guaiac-based faecal occult blood test (gFOBt) screening programme. The NHS Bowel Cancer Screening Programme (BCSP) in England began offering biennial gFOBt screening to men and women aged $60-69$ years $(\sim 10 \%$ of the population) in July 2006. The phased roll-out achieved national coverage in January 2010 and from 2008 the screening age range was extended to 74 years. An early analysis of the first 2.1 million subjects invited to screening showed an uptake of $55-60 \%$. Of all subjects tested, $2 \%$ were gFOBt positive and $8 \%$ had cancer (Rees and Bevan, 2013). From its inception to the end of May 2014, the BCSP had diagnosed 19045 cancers (personal communication BCSP National Office), with a higher proportion of Dukes' A cancers compared with non-screened patients (35\% vs 13\%) (Morris et al, 2012; Rees and Bevan, 2013). Currently, $3 \%$ of patients with CRC are diagnosed through the NHS BCSP (Morris et al, 2012) and is comparable to that reported for countries with similar health systems (Gatta et al, 2000; Lynch et al, 2007).

The BCSP uses the gFOBt (hema-screen; Immunostics, NJ, USA), which is designed to identify subjects at risk of colonic neoplasia by detecting intraluminal bleeding from vascularised CRCs and adenomas. The degree of blood loss is related to the size, stage and site of the neoplasia (Macrae and St John, 1982). Subjects perform the test at home by placing in turn two faecal samples from each of three separate stool sample onto a card with a total of six windows. The gFOBt test uses the pseudoperoxidase activity of haem (from haemoglobin) to release oxygen from hydrogen peroxide and convert colourless guaiac, impregnated in the test card, to a blue colour (Greegor, 1971).

The BCSP in England is co-ordinated by five regional Hubs; screening kits are returned to an accredited Hub laboratory where stool samples applied to all six windows are analysed by a manual qualitative process. A kit is reported as 'normal' if none of the six windows (spots) is positive. A kit with five or six positive spots is designated 'abnormal' and further investigation, usually colonoscopy, is recommended. A kit with between one and four positive spots is considered 'unclear' and a second kit is sent out to the subject. If the second kit result is normal, a third kit is sent out. If either the second or third kits contain one or more positive spots then the outcome is described as 'weak positive' and colonoscopy is recommended. Following an unclear result in kit one, if both kits are normal then patients are returned to the screening programme for repeat testing in 2 years' time (Figure 1).

Subjects with abnormal and weak positive test results are referred for colonoscopy. If this is normal, the subject is returned to the screening programme for a repeat gFOBt in 2 years' time. Subjects diagnosed with CRC are referred to their local CRC multidisciplinary team. If polyps or adenomas are found then surveillance follows the British Society of Gastroenterology guidelines (Cairns et al, 2010).

\section{Aims}

1. To determine the risk of a CRC or adenoma from the gFOBt positivity patterns, using a novel method based on spot positivity \% (SP\%).

2. To determine if the current SP algorithm can be improved to enable more effective use of a limited colonoscopy resource.

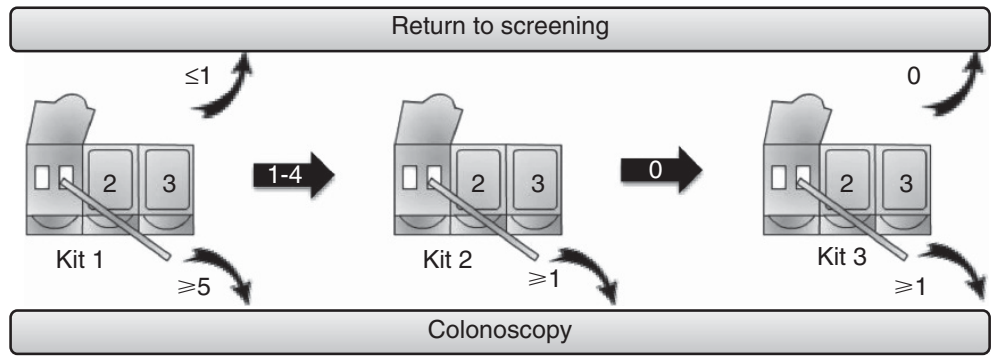

\begin{tabular}{|c|c|c|c|c|}
\hline \multirow[t]{2}{*}{ 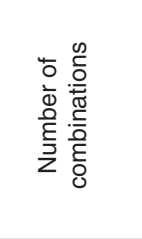 } & $\bullet$ & ๑๑॰ & 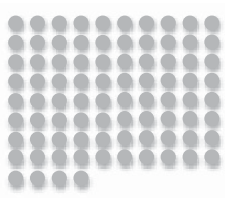 & $\bullet \bullet$ \\
\hline & 1 & 4 & 48 & 2 \\
\hline Description & - Normal & $\begin{array}{l}\text { - Kit } 1 \text { unclear } \\
\text { - Kit } 2 / 3 \text {-ve }\end{array}$ & $\begin{array}{l}\text { - Kit } 1 \text { unclear } \\
\text { - Kit } 2 / 3 \text { +ve }\end{array}$ & - Abnormal \\
\hline $\mathrm{SP} \%$ & $0 \%$ & $6-22 \%$ & $11-83 \%$ & $83-100 \%$ \\
\hline Outcome & Screening & Screening & Colonoscopy & Colonoscopy \\
\hline
\end{tabular}

Figure 1. This flowchart demonstrates how the different positivity patterns generated by the three kits create 55 positivity combinations. The BCSP algorithm deals with these combinations differently depending on the spot pattern and SP\%. There were subjects with an unclear kit one and normal results in kits two and three that are returned to the screening programme with a higher SP\% than some weak positive combinations leading to colonoscopy. These weak positive combinations derive from an unclear result in kit one followed by some positive spots in either kit two or three. 


\section{MATERIALS AND METHODS}

The Bowel Cancer Screening System database was used to identify all individuals from the BCSP Southern Hub with two consecutive episodes of 'adequate screening'. ('Adequate screening' is defined as a definitive normal or abnormal screening test result). Episode 1 occurred between April 2009 and March 2011 and is referred to as the 'prevalent' round or first screen, Episode 2 refers to the next round or first 'incident' round of screening.

Each test kit was coded with six-digit combinations of $\mathrm{N}$ (for negative test spot) or $\mathrm{P}$ (for a positive test spot). During the course of an 'adequately screened' episode up to three kits may be returned, each with six spots, providing a maximum of 18 spot results per episode. Overall, there were 55 individual spot combinations that map all the potential negative or positive results in the kits. For all combinations in episode 2 that refer participants for colonoscopy, we recorded both CRC and adenoma detection rates. The Programme currently records gFOBt outcomes as abnormal, weak positive or normal. In this study we used a new method of describing the gFOBt SP variable called the 'SP\%'. The SP\% was calculated for each of the 55 combinations by dividing the number of positive spots by the total number of spots returned during a single-screening episode. For example, currently a participant with a normal result has $0 / 6$ positive spots or an SP\% of $0 \%$. A participant with an abnormal result from having 5/6 positive spots has an SP\% of $83 \%$, whereas a weak positive result of $4 / 6,0 / 6$ and $1 / 6$ has a total of five positive spots out of a total of 18 , or therefore has an SP\% of $28 \%$.

For this study, individuals with designated abnormal (SP\% range from 83 to $100 \%$ ) or weak positive (SP\% range from 11 to $83 \%)$ results were referred to colonoscopy. Subjects with an unclear kit and one result followed by a normal kit two and three, had SP\%s of $6-22 \%$ and were returned to the screening programme (Figure 1). To investigate the possibility that neoplasia in individuals not currently referred for colonoscopy in Episode 1 could be missed, SP patterns at Episode 1 were analysed for individuals with cancer diagnosed at Episode 2.

Table 1. Demographic variables; including gender and age groups and the immediate outcomes following screening for Episode 2

\begin{tabular}{|c|c|}
\hline Variable & Episode 2, No. (\%) \\
\hline Number & 284261 \\
\hline \multicolumn{2}{|l|}{ Gender } \\
\hline Male & $130176(45.8)$ \\
\hline Female & $154085(54.2)$ \\
\hline \multicolumn{2}{|l|}{ Age group } \\
\hline$<60$ years & 0 \\
\hline 60-61years & $16(0.0)$ \\
\hline 62-63years & $50801(17.9)$ \\
\hline 64-65years & $62946(22.1)$ \\
\hline 66-67years & $60104(21.1)$ \\
\hline $68-69$ years & $55225(19.4)$ \\
\hline$\geqslant 70$ years & $55169(19.4)$ \\
\hline \multicolumn{2}{|l|}{ Screening outcome } \\
\hline Colonoscopy & 3891 (1.4) \\
\hline Not screened ${ }^{a}$ & $1272(0.4)$ \\
\hline Other diagnostic test & $122(0)$ \\
\hline Returned to screening & 278976 (98.1) \\
\hline \multicolumn{2}{|c|}{$\begin{array}{l}\text { In total, } 1.4 \% \text { of subjects underwent colonoscopy. } \\
\text { a This corresponds to the lost to follow-up. }\end{array}$} \\
\hline
\end{tabular}

The statistical analysis was conducted on anonymised data provided by the BCSP Southern Hub. No ethical approval was necessary. Patients scheduled for colonoscopy were consented following NHS guidelines.

SPSS (version 20.0; SPSS Inc., Chicago, IL, USA) was used for descriptive, $\chi^{2}$, linear and locally weighted scatterpoint smoothing (LOESS) non-linear regression analysis.

\section{RESULTS}

We studied 284261 individuals with a mean age at episode 1 of 63.8 years (s.d. 2.9); female subjects accounted for $54.2 \%$ $(n=154085)$ of the total (Table 1). Men (1.7\% (2193/130 176)) were more likely to have a gFOBt SP pattern, leading to a colonoscopy than females (1.1\% (1698/154 085)).

In episode 2, 3891 (1.4\%) individuals had a colonoscopy; 286 (7.4\%) were diagnosed with CRC, 7.5\% had high-risk, $13.1 \%$ intermediate-risk and 19.2\% low-risk adenomas (Table 2). Overall, $47.2 \%$ of episode 2 colonoscopies identified neoplasia.

Colonoscopy findings based on the gFOBt result; abnormal $v s$ weak positive. In episode 2, 90.6\% (3525/3891) of colonoscopies were performed following a 'weak positive' result, the remaining 9.4\% (366/3891) followed 'abnormal' results. Colorectal cancer was detected more frequently in colonoscopies performed following an abnormal gFOBt result $(21.3 \%$ vs $5.9 \%, P<0.001)$. There was no significant difference in the detection rate for all neoplasia (CRC and adenomas) between 'abnormal' and 'weak positive' gFOBt results $(51.9 \%$ vs $46.7 \%, P=0.06)$, which reflected the similar detection rates for adenomas between abnormal and weak positive gFOBt results (Table 2).

CRC risk based on the SP percentages (SP\%). There were 16 separate SP\%s, which ranged from 11 to $100 \%$ and led to a colonoscopy referral. The SP\% of $17 \%$ was the most common, accounting for $21.8 \%$ of colonoscopies. Consistent with the finding that most colonoscopies are performed after weak positive gFOBts, SP\%s of $\leqslant 25 \%$ accounted for $53.1 \%$ of all colonoscopies performed (Table 3).

Increasing SP\% was associated with an increase in the CRC detection rate. In episode 2 , at an SP\% of $11 \%$, the CRC detection rate was $4.2 \%$. At an SP\% of $100 \%$, CRC detection had increased to $24.5 \%$ (Figure 2). Between the two extremes, the CRC detection rate broadly increased linearly with an $R^{2}$ correlation (linear) of 0.89 .

A LOESS curve for non-linear regression modelled the risk of CRC at different SP\%s (Figure 3). At low SP\%s, of $11-25 \%$, the CRC risk was $\sim 4 \%$. Within this range, lies the SP pattern $4-0-0$ (created by four positive spots in kit one and normal results for kits two and three), with an SP\% of $22 \%$. Subjects with this pattern are currently not referred for colonoscopy. Above an SP\% of 25\%, the

\begin{tabular}{|c|c|c|c|c|}
\hline Episode 2 & $\begin{array}{c}\text { Total } \\
(n=3891), \\
\text { no }(\%)\end{array}$ & $\begin{array}{c}\text { Abnormal } \\
(n=366), \\
\text { no (\%) }\end{array}$ & $\begin{array}{c}\text { Weak } \\
\text { positive } \\
(n=3525), \\
\text { no }(\%)\end{array}$ & $P$-value \\
\hline Cancer & $286(7.4)$ & $78(21.3)$ & $208(5.9)$ & $<0.001$ \\
\hline Adenomas high risk & $290(7.5)$ & $20(5.5)$ & $270(7.7)$ & 0.13 \\
\hline Intermediate risk & $511(13.1)$ & $33(9.0)$ & $478(13.6)$ & 0.01 \\
\hline Low risk & $749(19.2)$ & $59(16.1)$ & $690(19.6)$ & 0.10 \\
\hline All neoplasia & $1836(47.2)$ & $190(51.9)$ & $1646(46.7)$ & 0.06 \\
\hline Normal & 2055 (52.8) & $176(48.1)$ & 1879 (53.3) & \\
\hline
\end{tabular}


Table 3. The frequency of colonoscopies performed for the different spot positivity percentages (SP\%)

\begin{tabular}{|l|c|c|}
\hline SP\% (Episode 2) & No (\%) & Cumulative, no (\%) \\
\hline $11 \%$ & $286(7.4)$ & $286(7.4)$ \\
\hline $17 \%$ & $847(21.8)$ & $1133(29.1)$ \\
\hline $22 \%$ & $241(6.2)$ & $1374(35.3)$ \\
\hline $25 \%$ & $693(17.8)$ & $2067(53.1)$ \\
\hline $28 \%$ & $106(2.7)$ & $2173(55.8)$ \\
\hline $33 \%$ & $564(14.5)$ & $2737(70.3)$ \\
\hline $39 \%$ & $21(0.5)$ & $2758(70.9)$ \\
\hline $42 \%$ & $289(7.4)$ & $3047(78.3)$ \\
\hline $44 \%$ & $9(0.2)$ & $3056(78.5)$ \\
\hline $50 \%$ & $230(5.9)$ & $3286(84.5)$ \\
\hline $56 \%$ & $0(0)$ & $3286(84.5)$ \\
\hline $58 \%$ & $121(3.1)$ & $3407(87.6)$ \\
\hline $67 \%$ & $68(1.7)$ & $3475(89.3)$ \\
\hline $75 \%$ & $32(0.8)$ & $3507(90.1)$ \\
\hline $83 \%$ & $176(4.5)$ & $3683(94.7)$ \\
\hline $100 \%$ & $208(5.3)$ & $3891(100)$ \\
\hline The cumulative column showed that SP\%s $\leqslant 25 \%$ accounted for $53.1 \%$ of all colonoscopies. \\
\hline
\end{tabular}

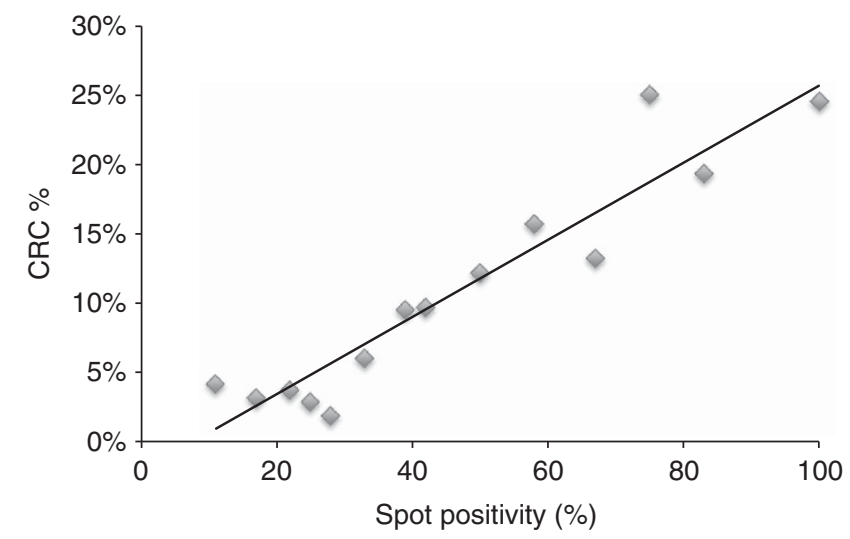

Figure 2. Correlation between spot positivity (SP\%) and CRC detection rate (\%) for gFOBT screening (Episode 2 of the BCSP, incident round). There is a linear relationship with $R^{2}=0.89(P<0.01)$.

CRC detection began to increase almost linearly; for every 10 -percentage points increase in SP\%, the CRC detection rate increased by $2.5 \%$ (Figure 3 ).

The gFOBt positive patterns have 55 combinations and include combinations that have 'unclear' in kit one followed by two normal kits $(n=4)$. These combinations had SP\% rates of $6-22 \%$ and are all currently returned for screening after 2 years, whereas the 48 combinations that are unclear in kit one and then contained one or more positive spots in either kit two or three (weak positives) had SP\%s of $11-83 \%$ and are all currently offered colonoscopy. There was one combination in the 'unclear then normal group' with an SP\% the same or higher than combinations in the unclear then positive group. This combination was 4-0-0 gFOBt and has an SP\% of $22 \%$ (Figure 3 ).

Spot positivity percentages and neoplasia (CRC and adenoma) risk. There was no correlation between SP\% and risk of any neoplasia (adenomas or CRC) $\left(R^{2}=0.09\right)$ (Figure 4).

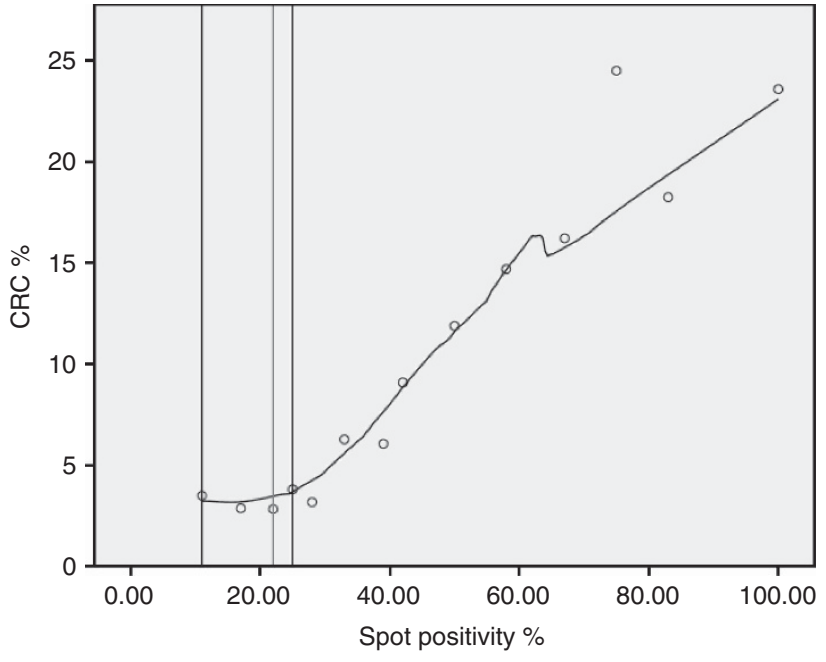

Figure 3. LOESS (locally weighted scatterpoint smoothing) non-linear regression modelling demonstrated that between an SP\% of 11-25\%, the CRC detection rate remained $\sim 4 \%$. Above an SP\% of $25 \%$, every 10 -percentage points increase was associated with a $2.5 \%$ increase in cancer. The vertical line drawn at an SP\% of $22 \%$, corresponds to the positivity pattern $4 \mathrm{NN}$ (created by four positive spots in kit one and normal results for kits two and three) a pattern not currently referred to colonoscopy.

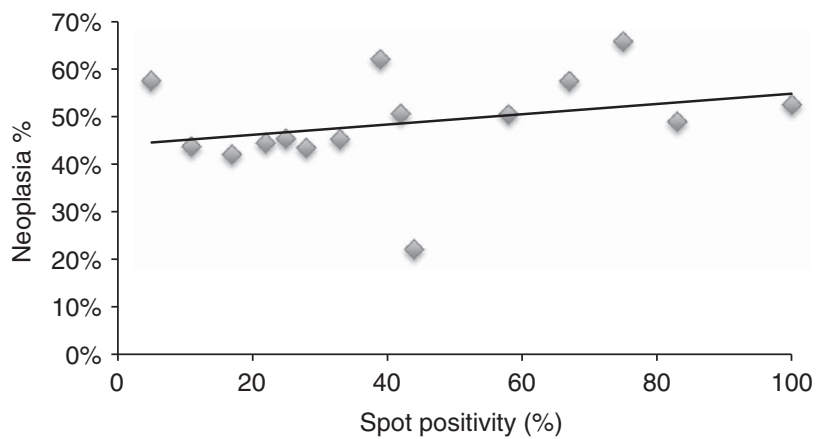

Figure 4. Correlation between spot positivity (SP\%) and overall neoplasia (cancer and adenomas) rate (\%) for gFOBT screening (Episode 2 of the BCSP, incident round). There is a poor linear relationship with $R^{2}=0.09$.

The association between SP\%, CRC detection and sex. Compared with females, male subjects were more likely to have a gFOBt combination that led to a referral for colonoscopy $(1.7 \%$ vs $1.1 \%)$ and were more likely to have CRC diagnosed at colonoscopy (8.3\% vs 6.2\%). Females had a lower risk of CRC detection at all SP\%s, whereas the linear correlation between SP\% and CRC was higher for males $\left(R^{2}=0.92\right)$ than females $\left(R^{2}=0.66\right)$ (Figure 5$)$.

Episode 1 gFOBt positivity patterns in subjects diagnosed with CRC in Episode 2. In episode 2, there were 286 subjects diagnosed with CRC, of whom 259 (90.6\%) had normal gFOBT results (i.e. had six negative spots) at episode 1 . The remaining 27 (9.4\%) subjects had some positive spots at episode 1 and four had colonoscopy, one patient had a low-risk adenoma and three had normal investigations. The remaining 23 CRC participants from episode 2 all had an unclear first kit followed by normal kits 2 and 3 at episode 1 (Figure 6). These subjects had SP\%s that ranged from 6 to $22 \%$.

Referral to colonoscopy of all individuals with an 'unclear' result for kit 1 (SP\%s 6-22\%) would identify all 23 cancers (or advanced 


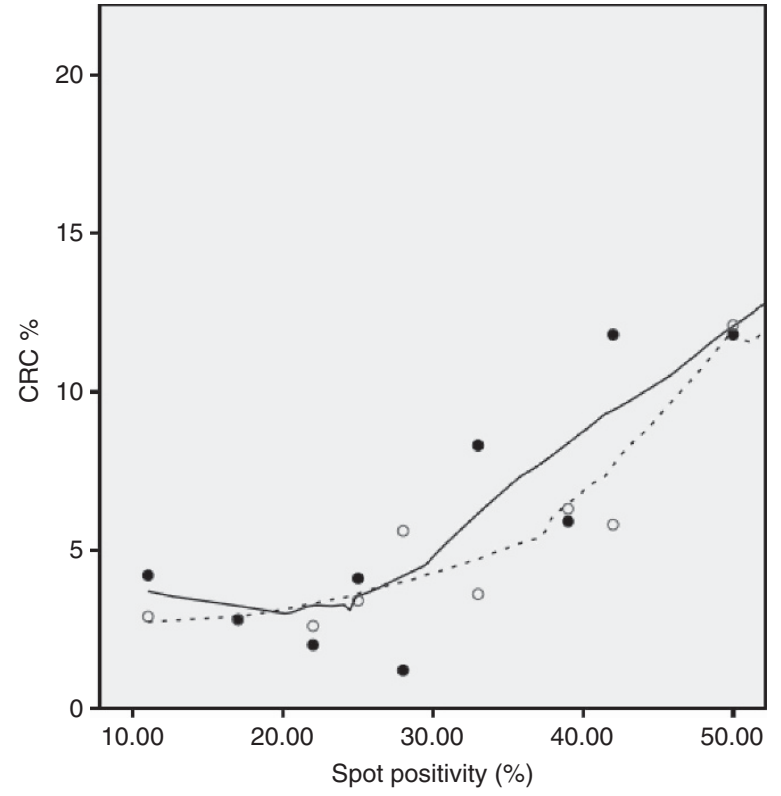

Figure 5. A LOESS curve demonstrates the relationship between SP\% and CRC detection rate for male (solid line) and female subjects (dotted line). The $R^{2}$ linear correlation in males was 0.92. However the CRC\% was $\sim 4 \%$ between an SP\% of $11-25 \%$ and only above an SP\% of $25 \%$ did the CRC\% increase lineally. In females, the $R^{2}$ linear correlation was 0.66 . The CRC\% remained below $5 \%$ from an SP\% of $11-35 \%$ and it is only when the SP\% was over 35\% that the CRC\% increased over $5 \%$.

adenoma) in episode 1 and would have required an additional 6115 colonoscopies with a CRC detection rate of only $0.4 \%$. If individuals with four positive spots on kit 1 (an SP\% of 22\%) had been referred for colonoscopy in episode 1, 229 additional colonoscopies would have been required with, potentially, five more cancers (or advanced adenoma) diagnosed, (detection rate $2.2 \%)$. In contrast, other spot combinations with an SP\% of $22 \%$ had a CRC detection of $4 \%$ (Figure 3). The resource implications of offering colonoscopy to all individuals with an SP\% of $22 \%$ would have required an additional 229 colonoscopies or $6 \%$ of the total colonoscopy workload.

\section{DISCUSSION}

This study provides a novel approach to evaluating the yield of gFOB testing by looking in detail at the gFOBt spot patterns and reveals the spot patterns of individuals that led to a diagnosis of CRC. Participants referred for colonoscopy on the basis of the current BCSP's algorithm have a CRC risk of $4-25 \%$. The increase in risk is broadly consistent with increasing gFOBt SP\%.

Most participants with an SP\%s of $11-100 \%$ are currently offered colonoscopy. At low levels of SP\% (11-25\%) the risk of CRC appears relatively constant at about $4 \%$. At higher SP\%s ( $>25 \%$ ) the risk of CRC increases linearly, so that for every $10 \%$ increase in the SP\%, the risk of CRC increases by $2.5 \%$. A previous study of NHS BCSP data showed the CRC risk was about 1 in 4 in the abnormal group, compared with 1 in 11 in the weak positive group (Lee et al, 2011). We have demonstrated similar findings, with a CRC rate in the abnormal group (21\%) equating to a one in five risk of CRC compared with a 1 in 17 risk (6\%) in the weak positive group. The results of this study show that not only is there a difference in risk between the two groups (abnormal or weak positive) but that there is a continuum of increasing risk with increasing SP\%.

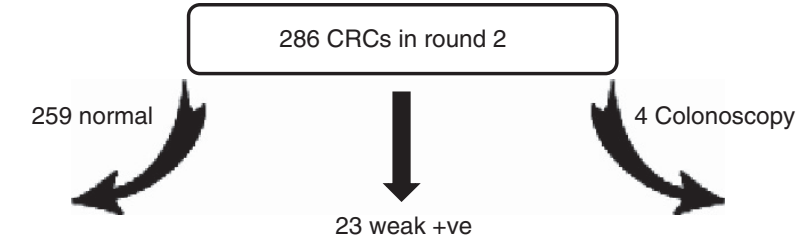

Options
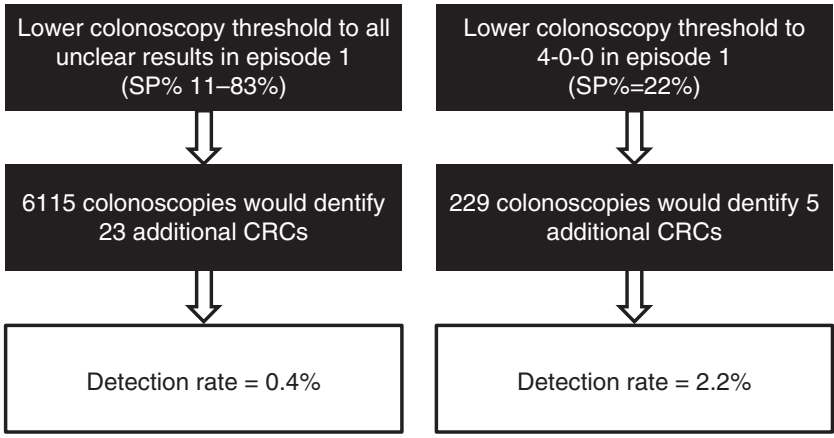

Figure 6. A flowchart that describes the gFOBt combinations in Episode 1 for individuals diagnosed with CRC following Episode 2. If all subjects with an unclear test result $(n=6115)$ received a colonoscopy, the CRC detection rate would be low (0.4\%). However, if only patients with an SP\% of $22 \%$ (following four positive spots in kit 1) were offered colonoscopy, then detection would be $2.2 \%$.

The increase in CRC risk with SP\% was not entirely linear. Between 11-25\% positivity, the risk remained at a fairly constant $4 \%$. Importantly, this group of subjects accounted for the majority (53.1\%) of colonoscopies performed in the study. The consistency of CRC rate at around $4 \%$ despite quite considerable change in the proportion of positive results is likely to be a limitation of the gFOBT. A systematic review found that the sensitivity of gFOBt for all neoplasms ranged from 6.2 to $83.3 \%$ and specificity from 65.0 to $99.0 \%$ (Burch et al, 2007). The low specificity is likely to be due to positive tests from non-cancer related GI bleeding and to analytical interference from dietary constituents. The high level of inter-study variability is due to differences in the study populations and test positivity definitions used. A meta-analysis of screening for CRC among asymptomatic individuals showed that repeated invitations to gFOBT screening, annually or biennially, reduced deaths from CRC by $16 \%$ (Hewitson et al, 2007). Despite this, gFOBT has a low clinical sensitivity and specificity, which means that many colonoscopies are scheduled for participants without pathology and some CRCs are missed owing to false-negative results. Our approach could not predict adenoma risk, even in patients with high-risk lesions. This has been shown in previous studies and demonstrates that while current gFOBt programmes can improve CRC survival they have not been shown to reduce CRC incidence through the accurate detection of pre-cancerous lesions. We have also shown that the correlation between SP\% and CRC was greater for males than females. The reasons for this correlation are not clear but it is consistent with previous reports of a significantly greater sensitivity of FOBt in men (Brenner et al, 2010). It may be that this is related to the higher rectal to colon ratio of cancers present in males (Massat et al, 2013).

This study has estimated the CRC risk for gFOBt positive spot combinations that do not currently lead to colonoscopy. This involved identifying subjects diagnosed with CRC in episode 2 and studying their episode 1 gFOBt combinations. We showed that subjects with four positive spots in kit one and normal results for kits two and three (4-0-0) in episode 1 , had a CRC risk of $2.2 \%$ at Episode 2. This is much higher than other combinations involving an unclear kit 1 , which had an overall CRC risk of $0.4 \%$, whereas 
lower than the CRC detection rate for subjects currently offered colonoscopy, which is $\sim 4 \%$. This may be an underestimate as this only reflects patients picked up in a further round of screening. The 4-0-0 combination does seem to be more risky than other unclear kit 1 combinations and the CRC rate is likely to be over $2.2 \%$ and around $4 \%$ as would be predicted from its SP\% of $22 \%$. This CRC risk is comparable with that of most subjects undergoing a BCSP colonoscopy. Additional evidence suggesting this group may be at increased risk comes from a study demonstrating that $7.8 \%$ of interval CRCs arise from gFOBts with an unclear result in kit one followed by two normal kits (Gill et al, 2012).

The effects of adjusting the NHS BCSP to offer colonoscopy to patients with a 4-0-0 combination need consideration because it is likely to produce a $6 \%$ increase in colonoscopy activity. In an average size endoscopy unit, covering a population of half a million, this would mean approximately two more lists a year (based on a 40-week year) (Nnoaham and Lines, 2008). Each colonoscopy (including pathology) currently costs $£ 245$. Assuming a $4 \%$ detection rate, each additional CRC diagnosis made would cost $£ 6125$, which is well within the willingness-to-pay threshold of $£ 20000$ currently used by NICE (Longworth et al, 2013).

This work has shown it may be possible to predict an individual's CRC risk more accurately, based on their gFOBt result. It must be borne in mind, however, that this is a screening programme so while these data may be used to advise patients about likely risks and benefits, advice must be set in the context of the wider role of screening and the importance of subsequent rounds. These results raise interesting questions about the information that should be provided to patients regarding their risk of a diagnosis of cancer.

Goyder et al, argue that when screening tests results are discussed with patients, there should be an attempt to not only discuss potential benefits and harms of further investigations but also the statistical chance of such events occurring (Goyder et al, 2000). Therefore, instead of delivering an abnormal ('positive') or normal ('negative') result, the focus is on a numeric scale, such as 1 in 20 or 1 in 4 chance of CRC. Ideally, in future, this would take account of more than simply the gFOBt result but factors such as age and sex. This idea is not novel, and numeric risk is well established for pregnant women having the 'triple test' for Down's syndrome (Fletcher et al, 1995). Information can be delivered to patients in many ways depending on preference. Individual risk can be described to subjects; numerically (the risk is 5\%), graphically (for example; 1 of 20 stick men on a page is differently coloured) or qualitatively (by comparing the risk with risky activities). The choice of tools used will depend on the subject's preferences, knowledge and needs (Bogardus et al, 1999) Shared decisionmaking between health professional and subjects using a personal risk assessment and decision aid appears to increase uptake of CRC screening (Schroy et al, 2012).

Traditionally, screening programmes have not sought to identify new understanding of disease, although they are, in fact, ideal ways of seeing how health care affects illness. Furthermore, it is rare for screening programmes to change in the face of new information gleaned from the programme (Bretthauer and Hoff, 2012). A highly organised programme such as the NHS BCSP provides an ideal data set for interrogation and analysis to refine and improve its own programme and others.

Limitations of the study included missing data on the site and stage of some of the CRCs, which prevented analysis of how these characteristics might affect the gFOBt result and SP\%. We also used an approximation of the CRC risk for gFOBt combinations that did not undergo colonoscopy. The clinical limitations of gFOBt (poor sensitivity and the development of disease in the period between episodes) is likely to have underestimated our risk estimates.
The current BCSP algorithm was based on the understanding that cancers bleed intermittently. This requirement means that in this programme, many subjects who have colonoscopy (except those with an abnormal kit one) need to have demonstrated bleeding in at least two kits some time apart. Our approach based on the SP\% is less dependent on recurrent bleeding over time. Testing our new methodological approach and in particular whether subjects with a 4-0-0 combination have a similar CRC risk to subjects already offered colonoscopy would require a change to the BCSP algorithm and prospective evaluation of the CRC detection rate in these patients.

The European guidelines for quality assurance in CRC screening and diagnosis concluded in 2010 that faecal immunochemical tests (FIT) offer substantially greater analytical sensitivity and specificity and allow enhanced detection of both cancer and adenomas. Since these guidelines were published, all countries commencing population screening have adopted FIT. The countries of the UK are now considering changing to FIT. A pilot study in Scotland has already shown that FIT improves uptake (Steele et al, 2013) and a pilot using FIT that commenced in England in April 2014 is also demonstrating markedly increased uptake from current gFOBt (BCSP Southern Hub report).

This paper explores the potential of determining risk from a binary test (spot positive or negative); FIT will provide a quantitative haemoglobin concentration that lends itself to the development of a multivariate risk score. The different sex-related sensitivities of blood as a biomarker for CRC and adenomas that have been highlighted in this paper can be exploited with FIT by applying different risk parameters for men and women. Longitudinal data from successive screening episodes that have been examined in this study shows great potential for enhancing risk prediction in any computerised population-based programme. Quantitative FIT will enable age, sex, screening history and even BMI, drinking, smoking and dietary history to be used in a way that is not easily achieved by gFOBt. However, it will take several years before FIT is rolled out, whereas the suggested changes to the screening programme could be adopted quickly and at minimal expense.

In conclusion, it is possible to demonstrate the risk of CRC arising from a particular gFOBt positivity pattern. The SP\% appears to predict CRC risk. Currently subjects with an SP\% of $11-100 \%$ are offered colonoscopy with CRC detection between 4 and $25 \%$. In subjects currently referred for colonoscopy, those with an SP\% of $11-25 \%$ have a CRC risk of $\sim 4 \%$. When the SP\% is $>25 \%$, for every $10 \%$ points increase, the CRC rate increases by $2.5 \%$. Consideration should be given to the merit of changing the current BCSP algorithm to address possible inequalities in detection of CRC risk (such as with 4-0-0 result). More detailed study of the risks, benefits and workload including health economics modelling should be undertaken. Additional consideration should be given to the information conveyed to subjects given the relative cancer risk of particular combinations.

ACKNOWLEDGEMENTS

Dr Helen Seaman's post as Research Fellow at the University of Surrey is funded by the Guildford Undetected Tumour Screening (GUTS) charity.

\section{REFERENCES}

Bernie T, Les I, Paul G, Jan K, David W, Chris S (1998) A systematic review of the effects of screening for colorectal cancer using the faecal occult blood test, Hemoccult'. BMJ 317(7158): 559-565.

Berrino F, De Angelis R, Sant M, Rosso S, Bielska-Lasota M, Coebergh JW, Santaquilani M (2007) Survival for eight major cancers and all cancers 
combined for European adults diagnosed in 1995-99: results of the EUROCARE-4 study. Lancet Oncol 8(9): 773-783.

Bogardus STJ, Holmboe E, Jekel JF (1999) Perils, pitfalls, and possibilities in talking about medical risk. JAMA 281(11): 1037-1041.

Brenner H, Haug U, Hundt S (2010) Sex differences in performance of fecal occult blood testing. Am J Gastroenterol 105(11): 2457-2464.

Bretthauer M, Hoff G (2012) Comparative effectiveness research in cancer screening programmes. BMJ 344: e2864.

Burch JA, Soares-Weiser St K, John DJB, Duffy S, Smith S, Kleijnen J, Westwood M (2007) Diagnostic accuracy of faecal occult blood tests used in screening for colorectal cancer: a systematic review. J Med Screen 14(3): 132-137.

Cairns SR, Scholefield JH, Steele RJ, Dunlop MG, Thomas HJW, Evans GD, Eaden JA, Rutter MD, Atkin WP, Saunders BP, Lucassen A, Jenkins P, Fairclough PD, Woodhouse CRJ (2010) Guidelines for colorectal cancer screening and surveillance in moderate and high risk groups (update from 2002). Gut 59(5): 666-689.

Coleman MP, Forman D, Bryant H, Butler J, Rachet B, Maringe C, Nur U, Tracey E, Coory M, Hatcher J, McGahan CE, Turner D, Marrett L, Gjerstorff ML, Johannesen TB, Adolfsson J, Lambe M, Lawrence G, Meechan D, Morris EJ, Middleton R, Steward J, Richards MA (2011) Cancer survival in Australia, Canada, Denmark, Norway, Sweden, and the UK, 1995-2007 (the International Cancer Benchmarking Partnership): an analysis of population-based cancer registry data. Lancet 377(9760): 127-138.

Fletcher J, Hicks NR, Kay JD, Boyd PA (1995) Using decision analysis to compare policies for antenatal screening for Down's syndrome. BMJ 311(7001): 351-356.

Gatta G, Capocaccia R, Sant M, Bell CM, Coebergh JW, Damhuis RA, Faivre J, Martinez-Garcia C, Pawlega J, Ponz de Leon M, Pottier D, Raverdy N, Williams EM, Berrino F (2000) Understanding variations in survival for colorectal cancer in Europe: a EUROCARE high resolution study. Gut 47(4): 533-538.

Gill MD, Bramble MG, Rees CJ, Lee TJW, Bradburn DM, Mills SJ (2012) Comparison of screen-detected and interval colorectal cancers in the Bowel Cancer Screening Programme. Br J Cancer 107(3pp. 417-421.

Goyder E, Barratt A, Irwig LM (2000) Telling people about screening programmes and screening test results: how can we do it better? J Med Screen 7(3): 123-126.

Greegor DH (1971) Occult blood testing for detection of asymptomatic colon cancer. Cancer 28(1): 131-134.

Hewitson P, Glasziou P, Irwig L, Towler B, Watson E (2007) Screening for colorectal cancer using the faecal occult blood test, Hemoccult. Cochrane Database Syst Rev (1): CD001216.

Lee TJW, Clifford GM, Rajasekhar P, Rutter MD, Kometa S, Ritchie MC, Waddup G, Nylander D, McNally RJQ, Rees CJ (2011) High yield of colorectal neoplasia detected by colonoscopy following a positive faecal occult blood test in the NHS Bowel Cancer Screening Programme. $J$ Med Screen 18(2): 82-86.

Longworth L, Youn J, Bojke L, Palmer S, Griffin S, Spackman E, Claxton K (2013) When does NICE recommend the use of health technologies within a programme of evidence development?: a systematic review of NICE guidance. Pharmacoeconomics 31(2): 137-149.

Lynch BM, Baade P, Fritschi L, Leggett B, Owen N, Pakenham K, Newman B, Aitken JF (2007) Modes of presentation and pathways to diagnosis of colorectal cancer in Queensland. Med J Aust 186(6): 288-291.

Macrae FA, St John DJ (1982) Relationship between patterns of bleeding and Hemoccult sensitivity in patients with colorectal cancers or adenomas. Gastroenterology 82(5 Pt 1): 891-898.

Massat NJ, Moss SM, Halloran SP, Duffy SW (2013) Screening and primary prevention of colorectal cancer: a review of sex-specific and site-specific differences. J Med Screen 20(3): 125-148.

Morris EJA, Whitehouse LE, Farrell T, Nickerson C, Thomas JD, Quirke P, Rutter MD, Rees C, Finan PJ, Wilkinson JR, Patnick J (2012) 'A retrospective observational study examining the characteristics and outcomes of tumours diagnosed within and without of the English NHS Bowel Cancer Screening Programme. Br J Cancer 107(5): 757-764.

Nnoaham KE, Lines C (2008) Modelling future capacity needs and spending on colonoscopy in the English bowel cancer screening programme. Gut 57(9): 1238-1245.

Rees CJ, Bevan R (2013) The National Health Service Bowel Cancer Screening Program: the early years. Expert Rev Gastroenterol Hepatol 7(5): 421-437.

Schroy PC, Emmons KM, Peters E, Glick JT, Robinson PA, Lydotes MA, Mylvaganam SR, Coe AM, Chen CA, Chaisson CE, Pignone MP, Prout MN, Davidson PK, Heeren TC (2012) Aid-assisted decision making and colorectal cancer screening: a randomized controlled trial. Am J Prev Med 43(6): 573-583.

Statistics, O. F. N. (n.d.)Office for National Statistics (2010) ed. Available at http://www.ons.gov.uk/ons/search/index.html?newquery= cancer + registrations.

Steele RJ, McDonald PJ, Digby J, Brownlee L, Strachan JA, Libby G, McClements PL, Birrell J, Carey FA, Diament RH, Balsitis M, Fraser CG (2013) Clinical outcomes using a faecal immunochemical test for haemoglobin as a first-line test in a national programme constrained by colonoscopy capacity. UEG 1(3): 198-205.

This work is published under the standard license to publish agreement. After 12 months the work will become freely available and the license terms will switch to a Creative Commons AttributionNonCommercial-Share Alike 3.0 Unported License. 